\title{
Analysis and Calculation for Sound Transmission Loss of Aircraft Fuselage Interior Panel
}

\author{
Han Feng ${ }^{+}$, Xu Junwei, and Feng Zixin \\ Senior Engineer, Shanghai Aircraft Research and Design Institute, Research on aeroacoustic and aircraft \\ acoustic design and noise \& vibration control, tel: 13918812293
}

\begin{abstract}
Combining acoustic theory of single wall and sandwich panel, the paper provided an analysis method to calculate sound transmission loss of typical aircraft fuselage interior panel. According to sound transmission loss equations, we defined the real parameters of interior panel and put them into calculation process, and then we got the result in flight and ground conditions. With comparing the data from calculation and measurement, we find that the value and trending could be marched well in special frequency range and there are drops at resonance, coincidence and dilatation frequencies of sandwich panel, and level of transmission loss is rising in flight condition.
\end{abstract}

Keywords: sound transmission loss, Sandwich panel, Fuselage interior panel

\section{Introduction}

As we all known, improving acoustic performance of aircraft cabin is improving the comfort for clients. Therefore, reducing the interior noise of cabin is one of trending for current commercial aircraft. But control cabin noise is not easy for engineering, especially for large aircraft, it is related the sound source from out side, e.g. engine and turbulence, sound transmitting of fuselage and absorption of interior furnish,

Normally, aircraft designers will do a lot of effort on improving the sound package attached on structure fuselage, such as interior panel, it is a main part of interior system for aircraft and important for insulating the exterior noise, so identifying and acquiring the sound transmission loss is necessary for aircraft acoustic design.

Generally, the interior panel is a sandwich plate made in composite material as seen in Fig. 1, consist of cover facing outside and core inside. As the development of aviation industry, the material of interior furnish is updated fast and fast, so how to quickly and accurately analysis and calculate sound transmission loss of interior panel is a key point for acoustic design in the early development phase.

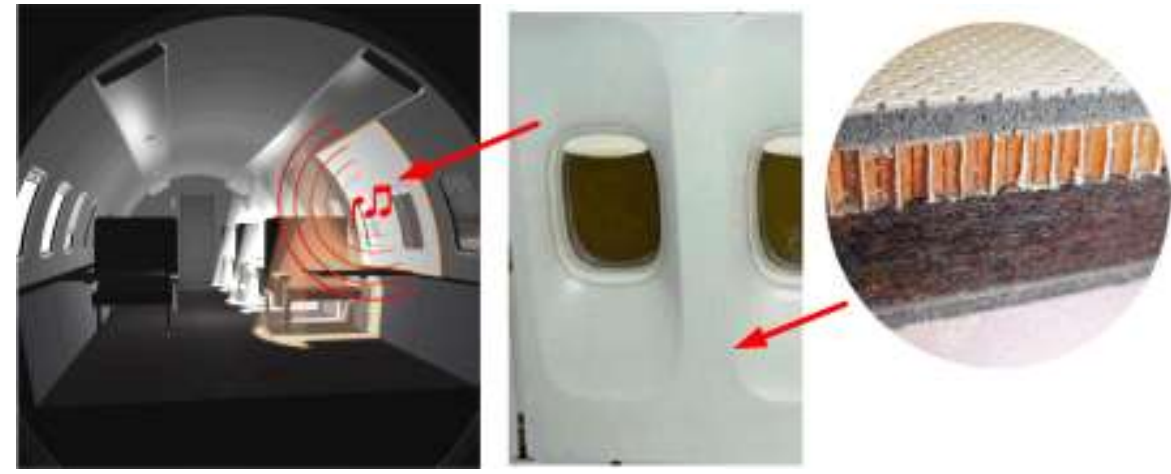

Fig. 1: Typical aircraft interior panel.

\footnotetext{
+ Corresponding author. Tel.: + 86 13918812293; fax: +86 20860270.

E-mail address: Hanfeng@ comac.cc.
} 
To find an engineering solution for issues above, this paper combined acoustic theory of single wall and sandwich panel, and provided an analysis method to calculate sound transmission loss of typical aircraft fuselage interior panel. Defining the real parameters of interior panel and put them into calculation process, we got the result in flight and ground conditions. Comparing the data from calculation and measurement, we checked the tolerance of the calculation methodology.

\section{Basic Theory}

\subsection{Single wall transmission loss}

When a plane sound wave hits a thin infinite flat plate, a part of the incident sound power will be reflected and a part will be transmitted through the plate.

The incident plane sound wave acts as a pressure field on the surface of the plate.

This pressure field causes the plate to deform perpendicular to the plate surface resulting in a bending wave in the plate.

The now vibrating plate on its turn introduces pressure variations in the air on both sides of the plate resulting in a sound wave on the incident side called the reflected sound wave and a sound wave on the other side called the transmitted sound wave.

During this process a part of the incident sound power will be reflected and absorbed by the plate, resulting in a transmitted sound power lower than the incident sound power.

The transmission loss is then defined by the following equation:

$$
T L=10 \log _{10}\left(\frac{P_{i n}}{P_{t r}}\right)=10 \log _{10}\left(\frac{1}{\tau}\right)
$$

In case we have air on both sides with same properties the equation for TL can be derived as follows:

$$
\tau=\frac{\left(2 \rho c_{s} / \omega m\right)^{2} \sec ^{2} \varphi}{\left[\left(2 \rho_{0} c_{s} / \omega m\right) \sec \varphi+\left(k / k_{b}\right)^{4} \eta \sin ^{4} \varphi\right]^{2}+\left[1-\left(k / k_{b}\right)^{4} \sin ^{4} \varphi\right]}
$$

According to reference 5:

$\mathrm{k}, \omega$ and $\phi$ represent respectively the wave number, the angular speed and the incidence angle of the incident sound wave

$\rho 0$ and cs are respectively the density and the speed of sound in air on both sides

$\mathrm{m}, \mathrm{kb}$ and $\eta$ represent respectively the mass per unit area of the plate, the free flexural wave number of the plate (dependent on the mass and stiffness of the plate) and the structure loss factor (a kind of damping constant of the plate, $\eta$ for elastic materials is of order 10-2-10-4).

The next figure (Fig. 2) shows the TL calculated with the equation (1).

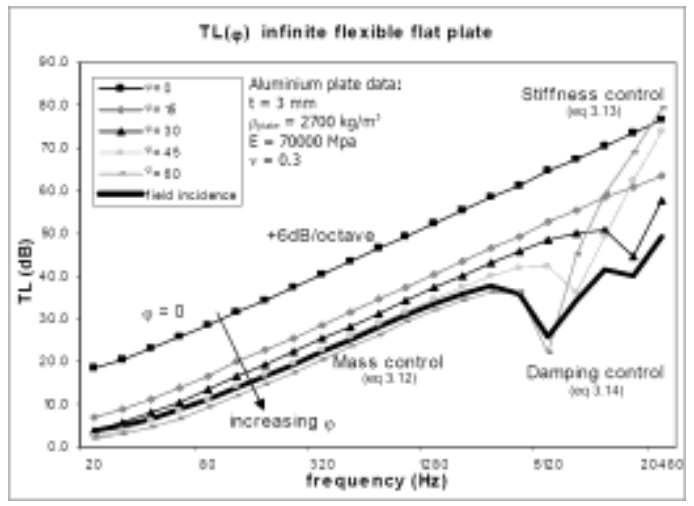

Fig. 2: Transmission loss of an infinite $3 \mathrm{~mm}$ thick aluminum plate for different fixed angles of incidence under diffuse incidence per reference [2]. 
The frequency where the TL shows a dip is called coincidence frequency and the reason is that the incident wave number coincides with the free flexural wave number of the plate.

The free flexural wave number of an infinite plate is given by the following expression:

$$
k_{b}=\sqrt[4]{\frac{\omega^{2} m}{D}}
$$

Where $\mathrm{D}$ is the bending stiffness per unit width of the plate with thickness $t$ and is expressed by:

$$
D=\frac{E t^{3}}{12\left(1-v^{2}\right)}
$$

When $k_{b}=k \sin \varphi$ for the coincidence we can derive the coincidence frequency as:

$$
f_{c}=\frac{1}{2 \pi} \sqrt{\frac{m}{D}}\left(\frac{c_{s}}{\sin \varphi}\right)^{2}
$$

The lowest coincidence frequency is called critical frequency $f_{c}$ and it occurs at $\varphi=90^{\circ}$

In practice sound waves are usually incident from many different angles simultaneously;

therefore the TL needs to be weighted for the different angle contributions:

$$
\tau_{d}=\int_{0}^{\pi / 2} \tau(\varphi) \sin 2 \varphi d \varphi
$$

The TL of an infinite plate under field incidence can then be derived based on Equation (2) and Equation (6) Below the coincidence frequency $(\mathrm{f}<<\mathrm{fc})$

Stiffness terms are relatively small compared to the mass terms.

$$
T L=20 \log _{10}\left(\frac{\omega m}{2 \rho_{0} c_{s}}\right)-10 \log _{10}\left(\ln \left\{1+\left(\frac{\omega m}{\rho_{0} c_{s}}\right)\right\}\right)
$$

Above the coincidence frequency $(\mathrm{f}>>\mathrm{fc})$

Mass terms are relatively small compared to the stiffness terms.

$$
T L=20 \log _{10}\left(\frac{\omega m}{2 \rho_{0} c_{s}}\right)+10 \log _{10} \eta+10 \log _{10}\left(\frac{2\left(1-\frac{f_{c}}{f}\right)}{\pi \frac{f_{c}}{f}}\right)
$$

At the coincidence frequency $(\mathrm{f}=\mathrm{fc})$

Damping term may not be neglected.

$$
T L=20 \log _{10}\left(\frac{\omega m}{2 \rho_{0} c_{s}}\right)+10 \log _{10} \eta
$$

As seen from these expressions the TL depends on the frequency, the material properties (such as density and elasticity) and the geometry (such as thickness). 


\subsection{Sandwich panel}

The single wall TL is characterized by the free flexural deformation mode while the TL for a sandwich panel is characterized by the combination of the free flexural deformation mode with the shear deformation mode. Moreover, if the core between the two faces is compressible, the dilatation modes will also affect the TL as Fig. 3.

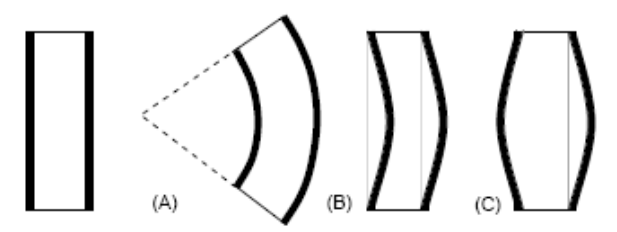

Fig. 3: flexural (a), shear (b), and dilatation (c) modes for a sandwich panel.

The sandwich panel will be described by a simplified empirical equation per reference [3]:

$$
T L=T L_{0}-10 \log \left[\frac{2 \sigma_{\text {radd }}}{\left(1-\left(\frac{f_{11}}{f}\right)^{2}\right)^{2} \cdot\left(1-\left(\frac{f}{f_{c}}\right)^{2}\right)^{2}+\eta_{e q}^{2}}+\frac{\pi \sigma^{2} f_{c}}{2 \eta f}\right]+10 \log \left[\left(1-\left(\frac{f}{f_{\text {dil }}}\right)^{2}\right)^{2}+\eta_{e q}^{2}\right]
$$

The first term refers to the mass law, the second term depends on the forced and resonant transmission while the last term is the dilatation response.

$$
T L_{0} \approx 20 \log \left(\frac{\omega m}{2 \rho_{0} c_{0}}\right) \text { is the mass law term. }
$$

The frequencies $\mathrm{f} 11, \mathrm{fc}$ and fdil can be expressed as:

$$
\begin{array}{r}
f_{11}=\frac{\pi}{2} \sqrt{\frac{D}{m}} \cdot\left[\left(\frac{1}{b}\right)^{2}+\left(\frac{1}{L}\right)^{2}\right] \\
f_{c}=\frac{c_{s}^{2}}{2 \pi} \sqrt{\frac{m}{D}} \\
f_{d i l}=\frac{1}{2 \pi} \sqrt{\frac{4 / 3 E_{c}}{t_{c}\left(2 m_{f}+m_{c} / 3\right)}}
\end{array}
$$

From the above expression following the subscript $f$ refers to the "face" of the sandwich panels while c refers to the "core" of the sandwich panel.

$$
\begin{array}{ll}
m=m_{c}+2 m_{f} & \text { Surface mass of the sandwich panel } \\
\mathrm{b}, \mathrm{L} & \begin{array}{l}
\text { Dimensions of plate } \\
\text { Sandwich stiffness }
\end{array} \\
D=\frac{1}{2} E_{f} t_{f}\left(t_{f}+t_{c}\right)^{2} & \text { Equivalent loss factor } \\
\eta_{\text {eq }}=\sqrt{\eta_{c}^{2}+0.1 \eta_{c}} & \text { Radiation coefficient } \\
\sigma_{\text {radd }} \cong \frac{1}{2}\left(0.2+\ln \left(2 \pi \frac{f}{c} \sqrt{S}\right)\right) &
\end{array}
$$




$$
\begin{aligned}
& \sigma_{r a d}=\frac{U c_{s}}{\pi^{2} S f_{c}^{3 / 2}} \sqrt{f} \\
& \sigma_{r a d}=0.45 \sqrt{\frac{U f_{c}}{c}} \\
& \sigma_{r a d}=1
\end{aligned}
$$

\title{
Radiation efficiency for $\mathrm{f}<<\mathrm{f}_{\mathrm{c}}$
}

\author{
Radiation efficiency for $\mathrm{f}=\mathrm{f}_{\mathrm{c}}$
}

Radiation efficiency for $\mathrm{f}>>\mathrm{f}_{\mathrm{c}}$

$\mathrm{S}$ and $\mathrm{U}$ are respectively the surface and perimeter of the panel of dimensions $\mathrm{b}$ and $\mathrm{L}$, while $\mathrm{m}$ is the mass per unit area.

$\mathrm{E}$ is the modulus of elasticity, $\eta \mathrm{c}$ is the loss factor of the core material and cs is the speed of sound in air.

The TL of a sandwich panel calculated using Equation (10) is given in the following Fig 4 and Fig 5 where three different panels are taken:

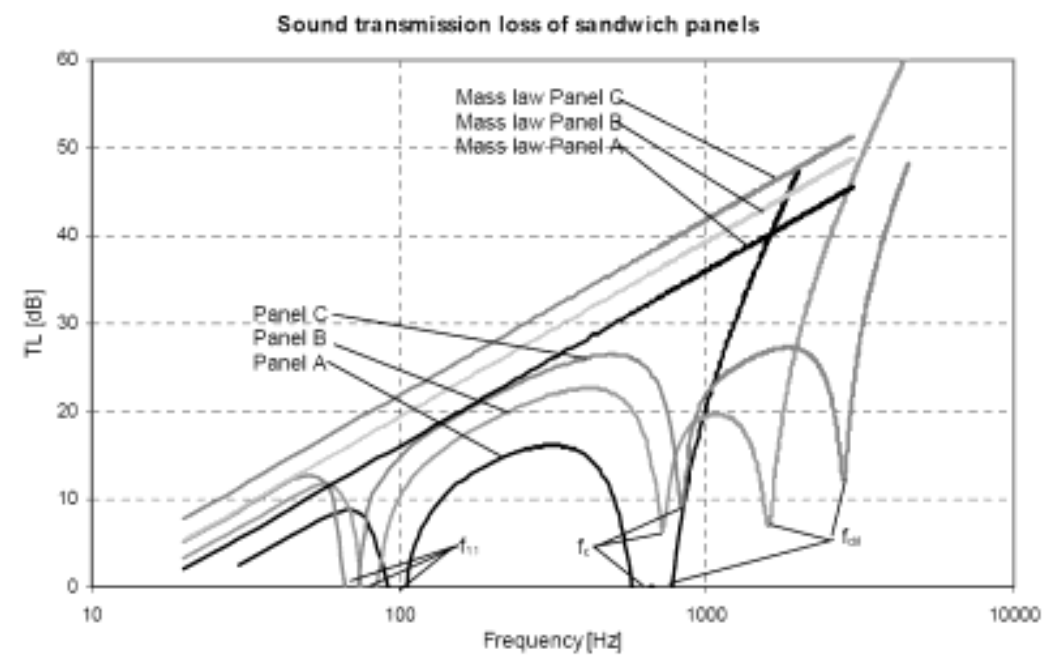

Fig. 4: Sound transmission of three $1 \mathrm{~m}^{2}$ sandwich panels.

\begin{tabular}{llll}
\hline & Panel A & Panel B & Panel C \\
\hline Facing: & & & \\
Thickness $(\mathrm{mm})$ & 4 & 4 & 4 \\
Density $\left(\mathrm{kg} / \mathrm{m}^{3}\right)$ & 800 & 800 & 800 \\
Elasticity $\left(\mathrm{N} / \mathrm{m}^{2}\right)$ & $1.3 \mathrm{e} 9$ & $1.3 \mathrm{e} 9$ & $1.3 \mathrm{e} 9$ \\
Poisson's ratio & 0.3 & 0.3 & 0.3 \\
Loss factor & 0.02 & 0.02 & 0.02 \\
\hline Core: & & & \\
Thickness $(\mathrm{mm})$ & 50 & 50 & 50 \\
Density $\left(\mathrm{kg} / \mathrm{m}^{3}\right)$ & 31 & 104 & 181 \\
Elasticity $\left(\mathrm{N} / \mathrm{m}^{2}\right)$ & $5.62 \mathrm{e} 6$ & $3.05 \mathrm{e} 7$ & $1.09 \mathrm{e} 8$ \\
Shear modulus $\left(\mathrm{N} / \mathrm{m}^{2}\right)$ & $1.2 \mathrm{e} 6$ & $8.7 \mathrm{e} 6$ & $3.02 \mathrm{e} 7$ \\
Poisson's ratio & 0.3 & 0.3 & 0.3 \\
Loss factor & 0.041 & 0.035 & 0.023 \\
\hline Dimensions & & & \\
Length $(\mathrm{m})$ & 1 & 1 & 1 \\
Width $(\mathrm{m})$ & 1 & 1 & 1 \\
\hline Characteristic frequencies & 97 & & \\
$\mathrm{f}_{11}(\mathrm{~Hz})$ & 741 & 1590 & 70 \\
$\mathrm{f}_{\text {dil }}(\mathrm{Hz})$ & 596 & 720 & 831 \\
$\mathrm{f}_{\mathrm{c}}(\mathrm{Hz})$ & & & \\
\hline
\end{tabular}

Fig. 5: Material properties of three $1 \mathrm{~m}^{2}$ sandwich panels.

\section{Analysis Process}

\subsection{Basic tool}

The equations of (1) to (10) provide a possible process to calculate the transmission loss of aircraft interior panel that is a type of sandwich panel. Based on the theory of 2 nd Chapter, we defined a tool by 
MATLAB Script to calculate the transmission loss of interior panel, such as follow fig 6, so that the analysis process of transmission loss interior panel were fixed in the program.

\subsection{Input properties}

As known from the analysis theory, the properties of interior panel need to be defined as input parameters for equations of calculation. Meanwhile, the environment performance around the interior system is also efficient factor for TL, especially, the air density and sound speed is difference between flight and ground condition. In the analysis process, following properties were provided for the interior side panel:

a) Environment Performance

1) Lab/Ground condition:

Air density $[\mathrm{kg} / \mathrm{m} 3]=1.225$;

Speed of sound $[\mathrm{m} / \mathrm{s}]=340$;

2) Flight condition:

Air density $[\mathrm{kg} / \mathrm{m} 3]=0.6756$;

Speed of sound $[\mathrm{m} / \mathrm{s}]=340$;

b) Facing properties of interior panel:

Density* $[\mathrm{kg} / \mathrm{m} 3]=1.800 \mathrm{E}+003$

Elastic modulus $[\mathrm{N} / \mathrm{m} 2]=1.900 \mathrm{E}+010$
Poisson coefficient $[/]=3.000 \mathrm{E}-001$

Thickness $[\mathrm{mm}]=3.000 \mathrm{E}-001$

Structural loss factor $[/]=1.000 \mathrm{E}-002$

Horizontal dimension $[\mathrm{m}]=1.060 \mathrm{E}+000$

Vertical dimension $[\mathrm{m}]=1.620 \mathrm{E}+000$

c) Core properties of interior panel:

Sandwich core density $[\mathrm{kg} / \mathrm{m} 3]=4.8 \mathrm{E}+001$

Sandwich core elastic modulus $[\mathrm{N} / \mathrm{m} 2]=5.5 \mathrm{E}+006$

Sandwich core thickness $[\mathrm{mm}]=6.0 \mathrm{E}+000$

Sandwich core structural loss factor $[/]=3.000 \mathrm{E}-002$

\subsection{Result of calculation}

Using the tool of chapter 3.1, according to Input properties of chapter 3.2, we can get the results of calculation about the transmission loss of interior panel. Transmission loss of interior panel in ground condition was compared with that of test data in lab provided a interior system supplier, as seen fig6. After seeing that calculation result is match well with test data in special frequency range, we can also get the TL curve of interior panel in flight condition as seen fig.7

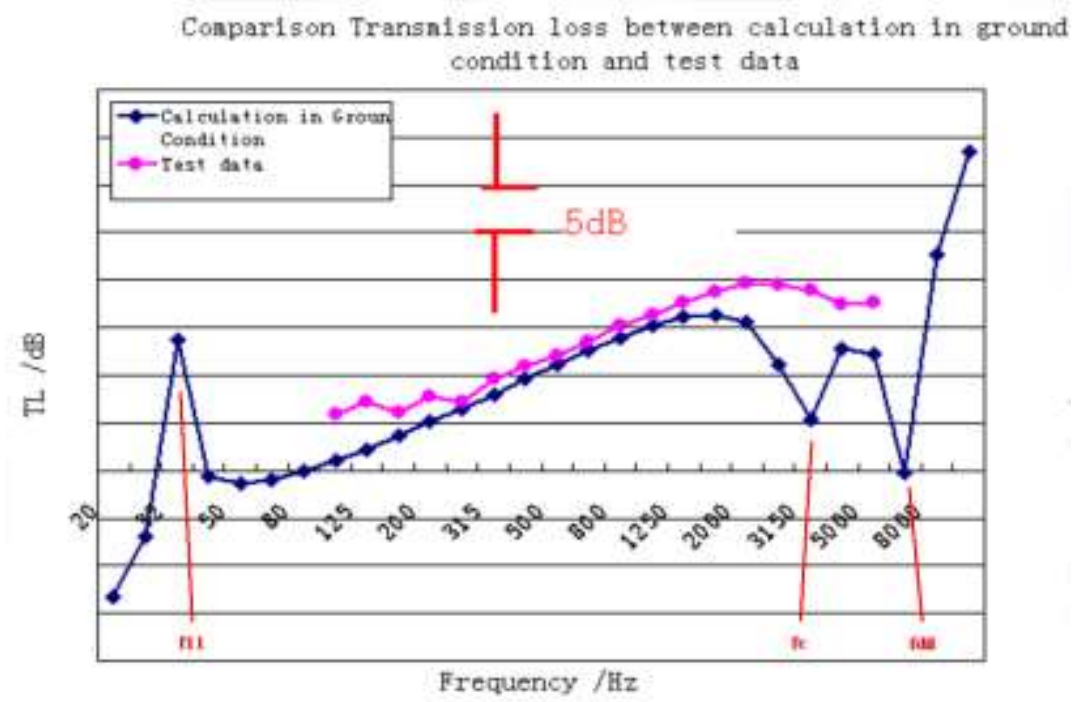

Fig. 6: TL calculation compared test data. 


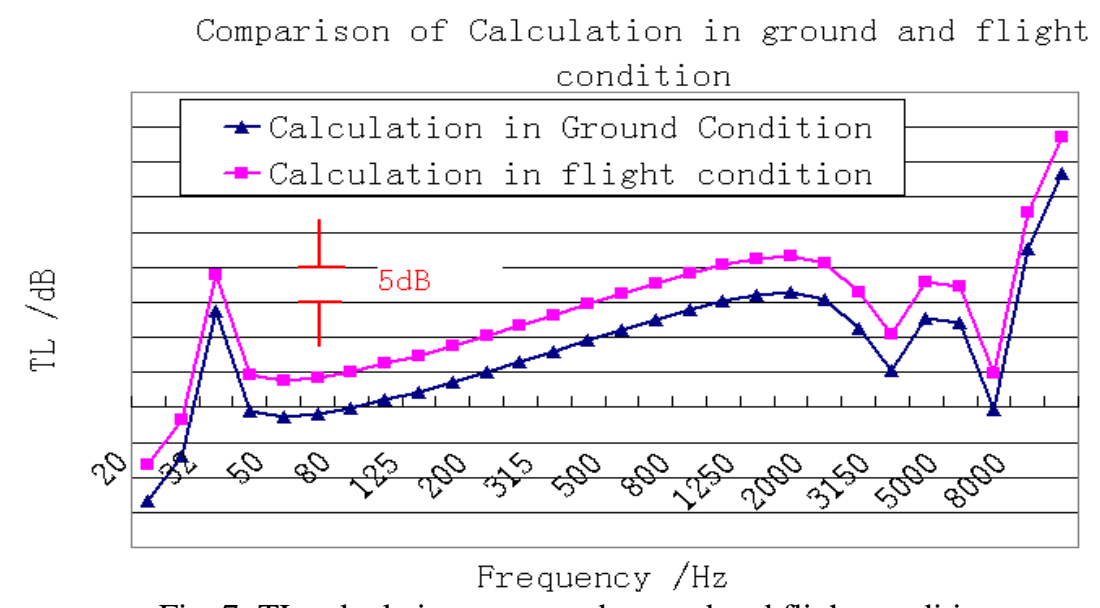

Fig. 7: TL calculation compared ground and flight condition.

\subsection{Result application}

According to the information of

Fig. 6, the level and tending of calculation is matching well with test data from $200 \mathrm{~Hz}$ to $2 \mathrm{kHz}$ and fitting for mass law above $8 \mathrm{kHz}$. So we have an enough confidence to apply this analysis method and program to calculate or predict the transmission loss of aircraft interior panel for acoustic design in development early stage.

Check the detail curve of transmission loss in fig.7, because of coincidence and dilation frequency, there are singularities (such as $\mathrm{f11}, \mathrm{fc}$, fdil) at the frequency range about from $3 \mathrm{kHz}$ to $8 \mathrm{kHz}$. The value of TL around these frequency ranges should be corrected by test data.

We can also found that for flight and lab condition, difference between two cases is about round $5 \mathrm{~dB}$. The reason is that the air density in aircraft cabin with flight condition is much smaller than that in ground and level of TL is rising in flight condition. This effect should be considered during prediction for sound pressure of aircraft cabin noise. However, this points need to research more and be supported by related measurement.

\section{Conclusion}

Combining acoustic theory of single wall and sandwich panel, the paper provided an analysis method to calculate sound transmission loss of typical aircraft fuselage interior panel and got the result in flight and ground conditions. With comparing the data from calculation and measurement, we find that the value and trending could be marched well in special frequency range and there are drops at resonance, coincidence and dilatation frequencies of sandwich panel. Meanwhile, level of transmission loss is rising in flight condition. Therefore, we can apply this analysis method and calculation program to predict sound transmission loss of interior panel in ground and flight condition for aircraft acoustic design with corrected by test data or experience database in early stage.

\section{References}

[1] Fahy, F., "Sound and structural vibration, radiation, transmission and response", Academic Press, London, 1989.

[2] Krakers,L.A., "Parametric fuselage design", Integration of mechanics and acoustic \& thermal insulation, $\mathrm{PhD}$ thesis TU Delft 2009

[3] Technical bulletin, "Acoustic behaviour of sandwich panels", DIAB group. 